Res., Soc. Dev. 2019; 8(5):e684712

ISSN 2525-3409 | DOI: http://dx.doi.org/10.33448/rsd-v8i4.712

\title{
Habilidades transversais de engenheiros em formação: o papel de projetos de extensão
}

Engineering students soft skill: the extension projects role

Las habilidades de travesía de ingenieros en la formación: el papel de proyectos de extensión universitária

Alana Santos Souza

ORCID: https://orcid.org/ 0000-0003-0309-4295

Universidade Federal de Itajubá, Brasil

E-mail: alana.sasouza@gmail.com

Lílian Barros Pereira Campos

ORCID: https://orcid.org/ 0000-0003-1657-7026

Universidade Federal de Itajubá, Brasil

E-mail: liliancampos@unifei.edu.br

Recebido: 03/11/2018 | Revisado: 03/12/2018 | Aceito: 12/02/2019 | Publicado: 13/02/2019

\section{Resumo}

A indissociabilidade entre ensino, pesquisa e extensão promove as atividades extensionistas ao mesmo patamar das demais responsabilidades universitárias. Isto é assegurado por lei e reafirmado por gestores do ensino superior. Diante disso, uma questão guiou esta pesquisa: "Qual o papel dos projetos de extensão no desenvolvimento de habilidades transversais (HT) de estudantes de engenharia?" Para responder esta questão, foi identificado na literatura 19 HT demandadas pelo mercado de trabalho no contexto da engenharia. Com base nisto, foi feito um levantamento on line, entre estudantes de engenharia da Universidade Federal de Itajubá - campus Itabira para identificar a frequiência que os projetos de extensão oportunizaram o uso destas HT. As respostas foram dadas em uma escala Likert que indicava 1 - nunca, 2 - raramente, 3- algumas vezes, 4- a maioria das vezes e 5 - sempre. 115 estudantes responderam o questionário e, analisando o percentual de respostas 4 (a maioria das vezes) e 5(sempre), foram identificadas as HT mais utilizadas: trabalho em equipe, flexibilidade, saber ouvir, comunicação e responsabilidade, com mais de $80 \%$ de respostas 4 e 5. As HT menos desenvolvidas foram argumentação e poder de persuasão, capacidade de aprendizagem e línguas estrangeiras, com menos de $40 \%$ de respostas 4 e 5 . Este artigo aponta evidências de que a extensão universitária é um processo educativo de prática de habilidades transversais. E, na educação na engenharia, isto é ainda mais importante uma vez 
que muitos acreditam que a sala de aula é um locus de desenvolvimento de habilidades exclusivamente técnicas.

Palavras-chave: extensão universitária; habilidades transversais; soft skills.

\section{Abstract}

The indissociability between teaching, research and extension promotes extension activities to the same level of other university responsibilities. This is ensured by law and reaffirmed by higher education managers. One question guided this research: "What is the role of extension projects in the development of transversal skills (TS) of engineering students?" To answer this question, 19 HTs were identified in the literature as demanded by the labor market in the context of engineering. Based on this, an online survey was carried out among engineering students from the Federal University of Itajubá - Itabira campus to identify the frequency that the extension projects provided the use of these TSs. Responses were given on a Likert scale that indicated 1 - never, 2 - rarely, 3 - sometimes, 4 - most times and 5 - always. 115 students answered the questionnaire and, by analyzing the percentage of answers 4 (most of the time) and 5 (always), the most used HT were identified: teamwork, flexibility, listening, communication and responsibility, with more than $80 \%$ of responses 4 and 5 . The less developed HTs were argumentation and power of persuasion, learning ability and foreign languages, with less than $40 \%$ of answers 4 and 5. This article indicates that university extension is an educational process of practice of transversal skills. And, in engineering education, this is even more important since many people believe that the classroom is a locus of exclusively technical skill development.

Keywords: university extension; transversal skills; soft skills.

\section{Resumen}

La indisociabilidad de la educación, investigación y extensión promueve las actividades de extensión al mismo nivel de las otras responsabilidades universitarias. Ante eso, una cuestión guió esta investigación: ¿¿Cual es el papel de los proyectos de extensión en el desarrollo de las habilidades transversales (HT) de estudiantes de ingeniería?" Para contestar a esa cuestión fue identificado en la literatura 19 HT demandadas pelo mercado de trabajo en el contexto de la ingeniería. Sobre esa base, fue hecho un levantamiento on line, entre estudiantes de ingeniería de la Universidade Federal de Itajubá - camous Itabira para identificar la frecuencia que los proyectos de extensión han permitido usar de estas HT. Las repuestas fueran dadas en una escala Likert que indicaba 1-nunca, 2-raramente, 3- algunas veces, 4- la mayoría de las 
veces y 5- siempre. 115 estudiantes contestaron lo cuestionario e, analizando el porcentual de respuestas 4(la mayoría de las veces) y 5(siempre), fueran identificadas las HT más utilizadas: trabajo en equipo, flexibilidad, saber escuchar, comunicación e responsabilidad, con mas de $80 \%$ de respuestas 4 y 5 . Las HT menos desarrolladas fueran argumentación y poder de persuasión, capacidad de aprendizaje y lenguas extranjeras, con menos de $40 \%$ de respuestas 4 y 5. Este artigo apunta evidencias de que la extensión universitaria es un proceso educativo de práctica de habilidades transversales. Y, en la educacion en la ingeniería, esto es aún mas importante una vez que muchos creen que las clases son lugares de desarrollo exclusivamente técnicas..

Palabras clave: extensión universitaria; habilidades transversales; soft skills.

\section{Introdução}

Novos imperativos sociais e econômicos tornam a educação para engenheiros cada vez mais importante. Engenheiros têm um papel decisivo no desenvolmento de tecnologias e inovação. Diante disso, faz-se necessário que as universidades assumam um papel de formar bons profissionais capazes de entrar e prosperar no local de trabalho (Täks, Tynjälä, Toding, Kukemelk \& Venessar, 2014). Este desafio torna-se maior quando Schulte (2004) diz que a universidade dos nossos tempos deve gerar criadores de emprego e não candidatos a emprego. Diante disso, escolas de engenharia encontram o desafio de fornecer aos estudantes uma ampla gama de habilidades e conhecimentos além do meramente técnico (Ohland, Frillman, Miller \& Carolina, 2004), incluindo habilidades transversais variadas (Torres, Velez Arocho \& Pabon, 1997).

Verdadeiramente, apenas os conhecimentos técnicos não estão sendo suficientes para o sucesso profissional. Assim surge o conceito de habilidades transversais (HT) que tratam-se de competências mais genéricas no mundo do trabalho, com atitudes e ações que ajudam o indivíduo a aplicar seus conhecimentos em situações diferentes, essenciais para a vida profissional. Para Mitchell \& Skinner \& White (2010), as HT são consideradas habilidades interpessoais que também podem ser denominadas soft skills.

Diante do desafio de desenvolver as HT de engenheiros em formação, esta pesquisa analisou como projetos de extensão contribuem para o desenvolvimento das HT em estudantes de engenharia. Como guia da pesquisa colocou-se a seguinte questão: "Qual o papel dos projetos de extensão no desenvolvimento de habilidades transversais (HT) de estudantes de engenharia?" Para responder esta questão, foi identificado na literatura 19 HT 
Res., Soc. Dev. 2019; 8(5):e684712

ISSN 2525-3409 | DOI: http://dx.doi.org/10.33448/rsd-v8i4.712

demandadas pelo mercado de trabalho no contexto da engenharia. Com base nisto, foi feito um levantamento on line, entre estudantes de engenharia da Universidade Federal de Itajubá campus Itabira para identificar a freqüência que os projetos de extensão oportunizaram o uso destas HT. O levantamento foi feito na Universidade Federal de Itajubá - campus Itabira. Segundo informado pela coordenação de extensão deste campus, há mais de 20 projetos de extensão em andamento que envolvem aproximadamente 500 estudantes dos 2500 matriculados neste campus. 115 estudantes responderam um questionário que possibilitou identificar o quanto as HT sob análise são desenvolvidas.

Este artigo está organizado em 5 partes, considerando esta primeira introdução. A segunda parte é a revisão da literatura sobre a importância dos projetos de extensão para o engenheiro em formação e sobre as habilidades transversais (HT) necessárias ao engenheiro em formação. Na terceira parte, foi colocado os procedimentos metodológicos e na quarta parte os dados analisados. Na quinta parte, foram colocadas considerações finais sobre o estudo e por fim as referências bibliográficas deste estudo.

\section{A importância dos projetos de extensão para o engenheiro em formação}

A atividade extensionista exerce um importante papel na formação discente e constitui-se como um importante resultado das instituições de ensino superior. Tanto é verdade que a Constituição de 1988 estabelece que ensino-pesquisa-extensão são indissociáveis (Brasil, 2009).

Esta questão é ratificada, na Lei de Diretrizes e Bases da Educação Nacional (Lei federal $\mathrm{n}^{\circ}$ 9.394, de 20 de dezembro de 1996), no artigo 46, inciso VII, que estabelece que uma das finalidades da Educação Superior é “promover a extensão, aberta à participação da população, visando à difusão das conquistas e dos benefícios resultantes da criação cultural e da pesquisa científica e tecnológica geradas na instituição" (Brasil, 1996). Isto coloca as atividade de extensão no mesmo patamar de importância que as preferidas atividades de ensino e pesquisa.

Segundo Puhl \& Dresch (2016) o princípio de indissociabilidade desta tríade possibilita novas formas pedagógicas de reprodução, produção e socialização de conhecimentos, efetivando a interdisciplinaridade. Para estes autores, por meio deste princípio é possível superar as dicotomias entre teoria/prática, sujeito/objeto e empiria/razão próprias do modo de pensar binário e linear predominante no meio acadêmico.

Como colocado pelo Fórum Nacional de Pró-Reitores de Extensão das Universidades Públicas Brasileiras (Forproex), "a extensão universitária é o processo educativo, cultural e 
científico que articula o ensino e a pesquisa de forma indissociável e viabiliza a relação transformadora entre a universidade e a sociedade" (Nogueira, 2000). Neste sentido, para além do conhecimento técnico obtido em sala de aula ou nos projetos de pesquisa, a atividade extensionista oportuniza o desenvolvimento e aplicação do conhecimento e ao mesmo tempo trabalha valores e atitudes cidadãs (Santos, 2012).

É interessante notar que a atividade extensionista, em grande parte, é organizada por projetos. Esta definição guarda um sentido importante destas atividades que, por acontecerem em formato de projetos, possibilitam um processo de aprendizagem ativa predominante no ensino da engenharia (Campos, Pinto \& Campos, 2018), que é o project based learning (PBL), ou seja, a aprendizagem baseada em projeto (Krajcik \& Blumenfeld, 2006) que é uma forma de aprendizagem situacional baseada nos achados construtivistas, na qual o estudante ganha uma profunda compreensão quando se envolve no desenvolvimento do conhecimento (Krajcik \& Blumenfeld, 2006).

O PBL vem ganhando espaço, especialmente nas universidades de ciências aplicadas, devido à necessidade do aluno de desenvolver diversas competências de aprendizagem para o ambiente profissional. É uma técnica que fornece experiências de aprendizagem multifacetadas, em oposição ao método de ensino tradicional (Lettenmeier, Autio \& Jänis, 2014).

Vários estudos provaram que o PBL é uma abordagem de aprendizagem ativa que pode ser organizada de várias maneiras. É importante mencionar que a PBL tem um impacto efetivo no desenvolvimento de habilidades transversais e, consequentemente, na formação profissional de estudantes de engenharia (Lima, Mesquita, Rocha \& Rabelo 2017; Van Hattum-Janssen \& Mesquita, 2011; Lima, Mesquita, Fernandes, Marinho-Araújo \& Rabelo 2015, Lima, Mesquita \& Flores 2014).

Considerando todas essas características do PBL, é possível perceber que as atividades de extensão carregam consigo possibilidades de aprendizagem que, muitas vezes, a sala de aula não contempla. Considerando o contexto específico da educação em engenharia, as atividades de extensão são a oportunidade dos estudantes aprenderem na prática comportamentos e conhecimentos técnicos de gestão e de outras áreas não contempladas nas grades de seus cursos.

\section{Habilidades Transversais (HT) necessárias ao engenheiro em formação}


ISSN 2525-3409 | DOI: http://dx.doi.org/10.33448/rsd-v8i4.712

Para este estudo foi utilizada a definição de habilidades transversais colocada por Mitchell \& Skinner \& White (2010) que são consideradas habilidades interpessoais que também podem ser denominadas soft skills. Analisando a literatura sobre o tema, foram identificadas 19 HT que também são denominadas transferíveis (Lima \& Mesquita \& Rocha \& Rabelo 2017), ou habilidades comportamentais (Robles, 2012; Ahmed \& Capretz \& Bouktif \& Campbell, 2013).

O Quadro 1 apresenta as 19 HT analisadas neste estudo a partir da literatura selecionada.

Quadro 1: Habilidades Transversais (HT) necessárias ao engenheiro em formação

\begin{tabular}{|c|c|c|c|}
\hline \# & $\begin{array}{l}\text { Habilidades } \\
\text { Transversais }\end{array}$ & Descrição & Autores \\
\hline 1 & $\begin{array}{l}\text { Atenção ao } \\
\text { detalhe }\end{array}$ & Saber observar os pequenos detalhes & $\begin{array}{l}\text { Direito \& Azevedo \& Pereira \& } \\
\text { Duarte, } 2012\end{array}$ \\
\hline 2 & $\begin{array}{l}\text { Argumentação e } \\
\text { Persuasão }\end{array}$ & $\begin{array}{l}\text { Conseguir expressar-se bem, expor } \\
\text { aos outros claramente suas ideias, } \\
\text { conquistar apoio e convencer a } \\
\text { aceitarem suas decisões ou } \\
\text { preferências. }\end{array}$ & $\begin{array}{l}\text { Direito \& Azevedo \& Pereira \& } \\
\text { Duarte, } 2012 \\
\text { Lima \& Mesquita \& Rocha \& Rabelo, } \\
2017\end{array}$ \\
\hline 3 & $\begin{array}{l}\text { Capacidade de } \\
\text { aprendizagem }\end{array}$ & $\begin{array}{l}\text { Ter capacidade de adotar novos } \\
\text { conhecimentos ou capacidade de ser } \\
\text { um eterno aprendiz }\end{array}$ & $\begin{array}{l}\text { Direito \& Azevedo \& Pereira \& } \\
\text { Duarte, } 2012\end{array}$ \\
\hline 4 & Comunicação & $\begin{array}{l}\text { Ter capacidade de comunicação oral } \\
\text { e escrita, além de saber escutar }\end{array}$ & $\begin{array}{l}\text { DIREITO \& AZEVEDO \& PEREIRA } \\
\text { \& DUARTE, 2012; CHAVES Et } \\
\text { Al.,2009; } \\
\text { LIMA \& MESQUITA \& ROCHA \& } \\
\text { RABELO, 2017; } \\
\text { ROBLES, 2012. }\end{array}$ \\
\hline 5 & $\begin{array}{l}\text { Criatividade e } \\
\text { inovação }\end{array}$ & Incentivar e apresentar novas ideias & $\begin{array}{l}\text { Direito \& Azevedo \& Pereira \& } \\
\text { Duarte, 2012; } \\
\text { Lima \& Mesquita \& Rocha \& Rabelo, } \\
2017 .\end{array}$ \\
\hline 6 & Flexibilidade & Ter capacidade de adaptação, & Direito \& Azevedo \& Pereira \& \\
\hline
\end{tabular}


ISSN 2525-3409 | DOI: http://dx.doi.org/10.33448/rsd-v8i4.712

\begin{tabular}{|c|c|c|c|}
\hline & & facilidade para mudanças, & $\begin{array}{l}\text { Duarte, 2012; } \\
\text { Chaves Et Al., } 2009 \\
\text { Robles, } 2012\end{array}$ \\
\hline 7 & Gestão do tempo & $\begin{array}{l}\text { Planejar o seu tempo de forma } \\
\text { consciente, analisando o tempo gasto } \\
\text { com atividades específicas e } \\
\text { cumprindo prazos }\end{array}$ & $\begin{array}{l}\text { Direito \& Azevedo \& Pereira \& } \\
\text { Duarte, 2012; } \\
\text { Chaves Et Al, } 2009\end{array}$ \\
\hline 8 & Liderança & $\begin{array}{l}\text { Ter capacidade de extrair o melhor } \\
\text { dos outros, agindo com sensatez e } \\
\text { influenciando pessoas }\end{array}$ & $\begin{array}{l}\text { Direito \& Azevedo \& Pereira \& } \\
\text { Duarte, 2012; } \\
\text { Lima \& Mesquita \& Rocha \& Rabelo, } \\
\text { 2017; }\end{array}$ \\
\hline 9 & $\begin{array}{l}\text { Línguas } \\
\text { estrangeiras }\end{array}$ & $\begin{array}{l}\text { Ter capacidade de se comunicar em } \\
\text { outros idiomas }\end{array}$ & $\begin{array}{l}\text { Direito \& Azevedo \& Pereira \& } \\
\text { Duarte, 2012; } \\
\text { Lima \& Mesquita \& Rocha \& Rabelo } \\
2017\end{array}$ \\
\hline 10 & Networking & $\begin{array}{l}\text { Buscar contato com outras pessoas } \\
\text { que trabalham no mesmo ramo }\end{array}$ & $\begin{array}{l}\text { Direito \& Azevedo \& Pereira \& } \\
\text { Duarte, 2012; }\end{array}$ \\
\hline 11 & Organização & $\begin{array}{l}\text { Manter em ordem seus afazeres e o } \\
\text { ambiente de trabalho }\end{array}$ & $\begin{array}{l}\text { Direito \& Azevedo \& Pereira \& } \\
\text { Duarte, 2012; } \\
\text { Chaves Et Al., 2009; Lima \& } \\
\text { Mesquita \& Rocha \& Rabelo, } 2017\end{array}$ \\
\hline 12 & $\begin{array}{l}\text { Orientação para } \\
\text { objetivos }\end{array}$ & $\begin{array}{l}\text { Ter capacidade de focar em } \\
\text { seus objetivos e trabalhar para } \\
\text { alcançá-los }\end{array}$ & $\begin{array}{l}\text { Direito \& Azevedo \& Pereira \& } \\
\text { Duarte, 2012; Lima \& } \\
\text { Mesquita \& Rocha \& Rabelo, } 2017\end{array}$ \\
\hline 13 & $\begin{array}{l}\text { Proatividade e } \\
\text { iniciativa }\end{array}$ & $\begin{array}{l}\text { Ter iniciativa e superar as } \\
\text { expectativas. Além disso, é tomar } \\
\text { atitudes antecipando um trabalho, ou } \\
\text { colhendo informações sobre } \\
\text { determinado assunto. }\end{array}$ & $\begin{array}{l}\text { Direito \& Azevedo \& Pereira \& } \\
\text { Duarte, 2012; Lima \& } \\
\text { Mesquita \& Rocha \& Rabelo, } 2017\end{array}$ \\
\hline & $\begin{array}{l}\text { Resolução de } \\
\text { problemas }\end{array}$ & $\begin{array}{l}\text { Ser capaz de resolver problemas o } \\
\text { mais prontamente e eficazmente } \\
\text { possível, muitas vezes recorrendo à } \\
\text { criatividade. }\end{array}$ & $\begin{array}{l}\text { Direito \& Azevedo \& Pereira \& } \\
\text { Duarte, 2012; } \\
\text { Chaves } \text { et al., 2009; }\end{array}$ \\
\hline
\end{tabular}


Res., Soc. Dev. 2019; 8(5):e684712

ISSN 2525-3409 | DOI: http://dx.doi.org/10.33448/rsd-v8i4.712

\begin{tabular}{|c|c|c|c|}
\hline 15 & Responsabilidade & $\begin{array}{l}\text { Responder pelos seus atos e pelas } \\
\text { obrigações que lhe foram atribuídas }\end{array}$ & $\begin{array}{l}\text { Direito \& Azevedo \& Pereira \& } \\
\text { Duarte, 2012; }\end{array}$ \\
\hline 16 & Saber ouvir & $\begin{array}{l}\text { Aceitar opiniões alheias, não } \\
\text { prejulgar o que está sendo dito e dar } \\
\text { atenção à quem fala. }\end{array}$ & $\begin{array}{l}\text { Direito \& Azevedo \& Pereira \& } \\
\text { Duarte, 2012; }\end{array}$ \\
\hline 17 & $\begin{array}{l}\text { Tolerância à } \\
\text { pressão }\end{array}$ & $\begin{array}{l}\text { Manter a calma e o foco em situações } \\
\text { surpresas e/ou desgastantes. }\end{array}$ & $\begin{array}{l}\text { Direito \& Azevedo \& Pereira \& } \\
\text { Duarte, 2012; } \\
\text { Chaves et al., 2009; }\end{array}$ \\
\hline 18 & $\begin{array}{l}\text { Tomada de } \\
\text { decisão }\end{array}$ & $\begin{array}{l}\text { Saber escolher um plano de ação } \\
\text { eficaz para uma determinada situação }\end{array}$ & $\begin{array}{l}\text { Direito \& Azevedo \& Pereira \& } \\
\text { Duarte, 2012; }\end{array}$ \\
\hline 19 & $\begin{array}{l}\text { Trabalho em } \\
\text { equipe }\end{array}$ & $\begin{array}{l}\text { Saber trabalhar com diferentes tipos } \\
\text { de pessoas, expondo suas opiniões e } \\
\text { sabendo escutar a dos demais. }\end{array}$ & $\begin{array}{l}\text { Direito \& Azevedo \& Pereira \& } \\
\text { Duarte, 2012; } \\
\text { Chaves } \text { et al., 2009; Lima \& } \\
\text { Mesquita \& Rocha \& Rabelo, } 2017 \\
\text { Robles, } 2012 .\end{array}$ \\
\hline
\end{tabular}

Na literatura selecionada, foram consideradas diferentes tipos de habilidades que são relevantes ao longo da formação de um engenheiro, pois estão sendo cada vez mais apreciadas e necessárias no mercado de trabalho. Estes estudos foram desenvolvidos já no contexto da engenharia apontando especificidades necessárias para engenheiros em formação.

Percebe-se que as situações profissionais que um engenheiro precisa enfrentar requisita uma série de comportamentos tornando desejável que tais HT sejam trabalhadas já na universidade. Quando considera-se que há um predomínio do método tradicional no ensino da engenharia (Campos \& Pinto \& Campos, 2018) surge um desafio urgente de se repensar práticas pedagógicas e processos educativos que possibilitem, não só a absorção de conhecimentos técnicos, mas também estas HT que são colocadas como importantes para o exercício da carreira de engenharia.

\section{Procedimentos metodológicos}

Para realização desta pesquisa foi realizado um levantamento quantitativo junto aos estudantes de graduação da Universidade Federal de Itajubá - campus Itabira. A UnifeiItabira foi fundada em 2008 e possui em torno de 2500 estudantes de nove cursos de Engenharia (Engenharia Mecânica, Engenharia da Mobilidade, Engenharia da Produção, Engenharia de Materiais, Engenharia de Saúde e Segurança, Engenharia Ambiental, 
Res., Soc. Dev. 2019; 8(5):e684712

ISSN 2525-3409 | DOI: http://dx.doi.org/10.33448/rsd-v8i4.712

Engenharia de Controle e Automação, Engenharia Elétrica e Engenharia da Computação). Este campus foi escolhido pela maior acessibilidade aos respondentes, por sua relevância comunitária e por possuir mais de 30 projetos de extensão registrados e mais de 500 estudantes envolvidos nestes projetos, conforme levantamento da Coordenação da Extensão da Unifei-campus Itabira.

Foi elaborado um questionário on-line estruturado com base nas 19 HT essenciais para o engenheiro em formação identificadas na literatura, publicado pelos pesquisadores nas redes sociais e plataforma educacional da Unifei. O instrumento de pesquisa teve o objetivo de identificar o quanto os estudantes percebem que suas habilidades transversais foram desenvolvidas e se, no âmbito do projeto, os estudantes tiveram oportunidade de usar estas habilidades. No questionário foram colocadas 19 afirmações sobre a oportunidade que os estudantes tiveram de usar estas HT durante o projeto. Uma escala Likert foi proposta para as respostas de modo que os estudantes manifestaram o quanto freqüente foram as situações colocadas, sendo 1 - Nunca, 2 - Raramente, 3 - Algumas Vezes, 4 - A maioria das vezes e 5 Sempre. Ao final do questionário foi colocado um espaço para que o respondente colocasse comentários e sugestões para a pesquisa. Os questionários foram aplicados em abril de 2018.

A partir da análise da freqüência com a qual cada situação de utilização das habilidades foi relatada, gerou-se uma avaliação quantitativa do quanto os projetos de extensão contribuem para o desenvolvimento de HT em engenheiros em formação. Assim, para a análise dos dados, foi considerado o percentual de respondentes que responderam 4 e 5 para as afirmações colocadas, declarando que as HT em questão foram utilizadas frequentemente durante o projeto.

\section{Resultados}

O questionário recebeu 115 respostas distribuídas de modo desigual entre os cursos ofertados na Unifei-campus Itabira. Conforme colocado na Tabela 1, houve uma quantidade maior de respondentes dos cursos de Engenharia da Produção, Engenharia Elétrica e Engenharia Mecânica. Estes dados não podem ser utilizados para analisar a participação de extensionistas por curso, uma vez que o método utilizado para a coleta de dados pode não ter sido eficaz para a coleta igualitária entre os cursos. De qualquer modo, este resultado aponta para investigações futuras que possam respaldar esta análise.

Tabela 1 - Quantidade de Respondentes por curso 
Res., Soc. Dev. 2019; 8(5):e684712

ISSN 2525-3409 | DOI: http://dx.doi.org/10.33448/rsd-v8i4.712

\begin{tabular}{l|r}
\hline \multicolumn{1}{c|}{ Curso } & Quantidade de Respondentes \\
\hline Engenharia Elétrica & 26 \\
\hline Engenharia de Produção & 22 \\
\hline Engenharia de Controle e Automação & 16 \\
\hline Engenharia Mecânica & 15 \\
\hline Engenharia Ambiental & 12 \\
\hline Engenharia de Computação & 10 \\
\hline Engenharia de Materiais & 6 \\
\hline Engenharia de Mobilidade & 6 \\
\hline Engenharia de Saúde e Segurança & 2 \\
\hline
\end{tabular}

Fonte: formulado pelas autoras

As respostas ao questionário foram relativas a 27 projetos de extensão registrados na Unifei-Itabira, conforme colocado na Tabela 2. Quanto ao tipo, foram identificados 7 projetos de difusão de ciência e tecnologia, 6 projetos de competições tecnológicas, 5 projetos de empresas juniores, 2 projetos culturais, 2 projetos de difusão do empreendedorismo, 2 projetos esportivos, 1 projeto social, 1 projeto de representação estudantil e 1 projeto de prática de idiomas.

Tabela 2 - Quantidade de Respondentes por projeto de extensão analisado

\begin{tabular}{c|c|c}
\hline Projeto & Tipo de Projeto & $\begin{array}{c}\text { Quantidade de } \\
\text { Respondentes }\end{array}$ \\
\hline Engenheiros sem Fronteiras & Social & 19 \\
\hline L.O.T.S Aero Design & Competições Tecnológicas & 13 \\
\hline Up Empresa Jr. & Empresa Júnior & 10 \\
\hline Equipe Mountain Baja & Competições Tecnológicas & 10 \\
\hline Iron Racers & Competições Tecnológicas & 8 \\
\hline Centro Acadêmico & Representação Estudantil & 6 \\
\hline Atlas Empresa Jr & Empresa Júnior & 5 \\
\hline Equipe de Robótica & Competições Tecnológicas & 5 \\
\hline Drumonsters & & 5 \\
\hline Quarta Arte & Cultural & 4 \\
\hline Startup Weekend & Empreendedorismo & \\
\hline Pet física & Difusão de Ciência e Tecnologia & \\
\hline
\end{tabular}


Res., Soc. Dev. 2019; 8(5):e684712

ISSN 2525-3409 | DOI: http://dx.doi.org/10.33448/rsd-v8i4.712

\begin{tabular}{c|c|c}
\hline Leste\&Negócios & Empreendedorismo & 3 \\
\hline Atlética & Esporte & 2 \\
\hline Bateria Calamgodum & Eultural & 2 \\
\hline Brick Jr. & Empresa Júnior & 2 \\
\hline Tetra Empresa Jr. & Esporte & 2 \\
\hline Cheerleaders & Empresa Júnior & 1 \\
\hline PET Ambiental & Difusão de Ciência e Tecnologia & 1 \\
\hline Desafio sampe & Difusão de Ciência e Tecnologia & 1 \\
\hline Dharma Empresa Jr & Difusão de Ciência e Tecnologia & 1 \\
\hline HighFive & Difusão de Ciência e Tecnologia & 1 \\
\hline Máquinas de Leonardo Da Vinci & Competições Tecnológicas & 1 \\
\hline Maratona de Programação & Difusão de Ciência e Tecnologia & 1 \\
\hline Maré Verde & Idiomas & 1 \\
\hline Segunda Língua & Difusão de Ciência e Tecnologia & 1 \\
\hline TEDX Unifei & Competições Tecnológicas & 2 \\
\hline Watermelon & &
\end{tabular}

Fonte: formulado pelas autoras

Pela análise dos tipos de projetos, percebe-se que há uma quantidade significativa de projetos de difusão de ciência e tecnologia, empresas juniores e competições tecnológicas. Ambos os projetos permitem que o estudante envolvido possa vivenciar situações profissionais que permitem que os envolvidos adquiram experiências importantes para seu preparo profissional. Vale salientar que as empresas juniores mencionadas são dos cursos de Engenharia da Produção (Up Empresa Jr.), Engenharia Ambiental (Dharma Empresa Jr.), Engenharia da Mobilidade (Brick Empresa Jr.), Engenharia de Controle e Automação (Atlas Empresa Jr.) e Engenharia de Materiais (Tetra Empresa Jr.). Este projetos, em específico, têm o objetivo de proporcionar aos seus integrantes a experiência em consultoria a empresas para solucionar problemas das respectivas áreas de conhecimento de seus cursos.

Já os projetos de competição tecnológica têm forte evolução de práticas de desenvolvimento e construção de veículos (Moutain Baja e Iron Racers), aeronaves (L.O.T.S Aero Design), jogos (Watermelon) e programação (Maratona de Programação). Os demais projetos têm objetivos variados nas áreas empreendedorismo, social, cultural, esportes, representação estudantil e idiomas. 
ISSN 2525-3409 | DOI: http://dx.doi.org/10.33448/rsd-v8i4.712

Para identificar o desenvolvimento das HT levou-se em consideração o percentual de estudantes que declararam alta freqüiência de utilização (4- maioria das vezes e 5- sempre) de HT nos projetos de extensão analisados. A Figura 1 apresenta estes dados.

Figura 1 - Percentual de estudantes que declararam alta freqüência de utilização de HT nos projetos de extensão analisados

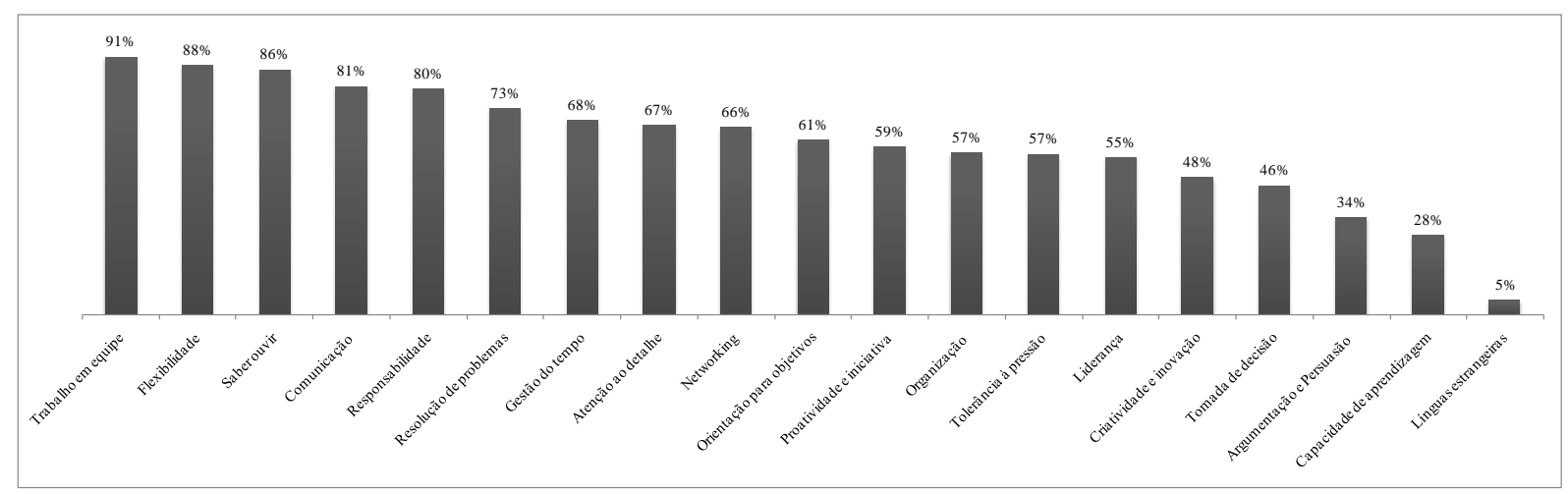

Fonte: formulado pelas autoras

As HT que merecem destaque são aquelas que mais de $77 \%$ declararam alta freqüência de utilização, a saber: Trabalho em equipe (91\%), Flexibilidade ( $88 \%)$, Saber ouvir (86\%), Comunicação (81\%), Responsabilidade (80\%) e Resolução de problemas (73\%). São estas as HT que os projetos analisados mais contribuíram para que os estudantes envolvidos nestes projetos desenvolvessem. Normalmente, a atividade extensionista envolve produções coletivas e exigem que os estudantes estejam em constante processo de adaptação a mudanças, solução de problemas, comunicação resultados e recebimento de feedback sobre seu trabalho.

Considera-se uma freqüência de utilização mediana, as que variaram entre $68 \%$ e $61 \%$ que foram as seguintes HT: Gestão do tempo (68\%), Atenção ao detalhe (67\%), Networking $(66 \%)$ e Orientação para objetivos (61\%). Estes resultados podem apontar oportunidades de melhoria nos projetos analisados para que sejam oportunizadas experiências para que os estudantes aprendam a gerir prazos e a fazer análise de ambiente no desenvolvimento de qualquer tarefa. Além disso, os dados apontam para a necessidade de os gestores de projetos de extensão atentarem-se para importância de se desenvolver a manutenção do foco e a construção de rede de contatos.

Para uma freqüência de utilização baixa foram consideradas as HT abaixo de $60 \%$ : Proatividade e iniciativa (59\%), Organização (57\%), Tolerância à pressão (57\%), Liderança 
Res., Soc. Dev. 2019; 8(5):e684712

ISSN 2525-3409 | DOI: http://dx.doi.org/10.33448/rsd-v8i4.712

(55\%), Criatividade e inovação (48\%), Tomada de decisão (46\%) e Argumentação e Persuasão (34\%), Capacidade de aprendizagem (28\%) e Línguas estrangeiras (5\%).

Diante deste resultado é possível perceber que ainda há HT relevantes pouco desenvolvidas nos projetos de extensão selecionados. Isto indica que é necessária a reflexão de como estes projetos são organizados e como os seus participantes organizam seu processo de desenvolvimento durante o mesmo.

\section{Considerações finais}

Este artigo foi conduzido pela seguinte questão: "Qual o papel dos projetos de extensão no desenvolvimento de habilidades transversais (HT) de estudantes de engenharia?". Foi identificado que os projetos analisados na UNIFEI - campus Itabira cumprem o papel de desenvolver 19 HT em medidas diferentes. Com destaque para a HT Trabalho em equipe, a mais desenvolvida e a HT Línguas Estrangeiras, a menos desenvolvida nos casos analisados.

O estudo aponta importantes reflexões para os extensionistas desta instituição e também oportunidades de estudos futuros para compreender ainda mais esta questão. Este artigo aponta que, em investigações futuras, deve-se analisar os projetos especificamente e analisar alguns projetos que melhor desenvolvem HT e verificar como as atividades são organizadas para fins de identificação de melhorias para os demais projetos.

\section{Agradecimentos}

Agradecemos todos os estudantes que responderam o questionário e à Coordenação de Extensão da Unifei - campus Itabira por ceder dados para o desenvolvimento da pesquisa.

\section{REFERÊNCIAS}

Ahmed, F. \& Capretz, L.F. \& Bouktif, S. \& Campbell, P. (2013). Soft Skills and Software Development: A Reflection from Software Industry. International Journal of Information Processing and Management (IJIPM), 4 (3),171-191.

Campos, Lilian Barros Pereira \& Pinto, Janaina Antonino \& CampoS, Roger Júnio.(2018). Entrepreneurial and Engineering Education - a twofer proposal. In: 9th International Symposium on Project Approaches in Engineering Education (PAEE) 15th Active Learning in Engineering Education Workshop (ALE).Anais. Brasília. 
Chaves, António \& Basto, Diogo \& Teixeira, Diogo \& Pacheco, Guilherme; Cruz \& Henrique; Costa \& João; Pires \& Nuno.(2009). Soft Skills: Enriquecimento do Curriculum Vitae. (2009). 35f. Trabalho realizado no âmbito da disciplina Projecto FEUP, Faculdade de Engenharia da Universidade do Porto, Porto.

Direito \& I., Axevedo \& G., Pereira, A. \& Duarte, A. M. O. (2009).Competências transversais nas engenharias: comparação de estudantes do Brasil e Portugal. XL Congresso Brasileiro de Educação em Engenharia (Cobenge) Anais. Belém.

Krajcik, J. S. \& Blumenfeld, P. (2006). Project-based learning. In R. K. Sawyer (Ed.), The Cambridge handbook of the learning sciences. New York: Cambridge. 317-334.

Lettenmeier, M. \& Autio, S. \& Jänis, R. Project-based learning on life-cycle management - A case study using material flow analysis. Retrieved from: https://www.lamk.fi/projektit/ecomill/ecomill-esilla/Documents/WRFartikkeli\%20Kulinaaritalo.pdf

Lima, E. \& Lopes, R. M. \& Nassif, V. \& Da Silva, D.(2015). Opportunities to Improve Entrepreneurship Education: Contributions Considering Brazilian Challenges. Journal of Small Business Management, 53 (4),1033-1051.

Lima, R. M. \& Mesquita, D \&, Fernandes, S. \& Marinho-Araújo, C.\& Rabelo, M. (2015) Modelling the Assessment of Transversal Competences in Project Based Learning. In Fifth International Research Symposium on PBL, International Joint Conference on the Learner in Engineering Education (IJCLEE 2015), Anais. San Sebastian.

Lima, R. M. \& Mesquita, D. \& Flores, M. A. (2014) Project Approaches in Interaction with Industry for the Development of Professional Competences. Industrial and Systems Engineering Research Conference (ISERC 2014),Anais. Montréal.

Lima, R. M. \& Mesquita, D. \& Rocha, C.\& Rabelo, M. (2017) Defining the Industrial and Engineering Management Professional Profile: a longitudinal study based on job offers. Production Journal,27. doi: 10.1590/0103-6513.229916 
Mitchell, G. W. \& Skinner, L. B. \& White, B. J. (2010). Essential Soft Skills for success in the twenty-first century workforce as perceived by business educators. Delta Pi Epsilon Journal, 52 (1), 43-53.

Nogueira, Maria das Dores Pimentel (org). (2000). Extensão Universitária: diretrizes conceituais e políticas - Documentos básicos do Fórum Nacional de Pró-Reitores de Extensão das Universidades Públicas Brasileiras 1987 - 2000. Belo Horizonte: PROEX/UFMG; o Fórum.

Puhl, M. J. \& Dresch, O. I. (2016). O Princípio da Indissociabilidade entre Ensino, Pesquisa e Extensão e o Conhecimento. Revista Di@logus, 5 (1), 37-55.

Ohland, M. W. \& Frillman, S. A. \& Miller, T. K. \& Carolina, N. NC.(2004). State's Engineering Entrepreneurs Program in the Context of US. Education that Works: The NCIIA 8th Annual Meeting, 155-164.

Robles, M. M. (2012) Executive perceptions of the top 10 soft skills needed in today's workplace. Business Communication Quarterly, 75(4),453-465.

Santos, M. P. Extensão Universitária: Espaço de Aprendizagem Profissional e suas Relações com o ensino e a Pesquisa na Educação Superior.(2012). Revista Conexão UEPG, 8 (2),154163.

Täks, M. \& Tynjälä, P. \& Toding,M. \& Kukemelk, H. \& Venessar, U. (2014) .Engineering Students' Experiences in Studying Entrepreneurship. Journal of Engineering Education, 4 (103), 573-598.

Torres, M. A. \& Velez Arocho, J. I. \& Pabon, J. A.(1997). BA 3100 - Technology-Based Entrepreneurship: An Integrated Approach to Engineering and Business Education, 738743.Retrieved from: https://ieeexplore.ieee.org/abstract/document/635925/ 
ISSN 2525-3409 | DOI: http://dx.doi.org/10.33448/rsd-v8i4.712

Van Hattum-Janssen, N. \& Mesquita, D. (2011). Teacher perception of professional skills in a project-led engineering semester. European Journal of Engineering Education, 36 (5), 461 472.

\section{Porcentagem de contribuição de cada autor no manuscrito}

Alana Santos Souza - 60\%

Lílian Barros Pereira Campos - 40\% 